\title{
The dynamics of charged dust in magnetized molecular clouds
}

\author{
Hyunseok Lee, ${ }^{1 \star}$ Philip F. Hopkins ${ }^{1}$ and Jonathan Squire ${ }^{1,2}$ \\ ${ }^{1}$ TAPIR, Mailcode 350-17, California Institute of Technology, Pasadena, CA 91125, USA \\ ${ }^{2}$ Walter Burke Institute for Theoretical Physics, Pasadena, CA 91125, USA
}

Accepted 2017 May 4. Received 2017 April 9; in original form 2016 December 15

\begin{abstract}
We study the dynamics of large, charged dust grains in turbulent giant molecular clouds (GMCs). Massive dust grains behave as aerodynamic particles in primarily neutral dense gas, and thus are able to produce dramatic small-scale fluctuations in the dust-to-gas ratio. Hopkins \& Lee directly simulated the dynamics of neutral dust grains in supersonic magnetohydrodynamic turbulence, typical of GMCs, and showed that the dust-to-gas fluctuations can exceed factor $\sim 1000$ on small scales, with important implications for star formation, stellar abundances and dust behaviour and growth. However, even in primarily neutral gas in GMCs, dust grains are negatively charged and Lorentz forces are non-negligible. Therefore, we extend our previous study by including the effects of Lorentz forces on charged grains (in addition to drag). For small-charged grains (sizes $\ll 0.1 \mu \mathrm{m}$ ), Lorentz forces suppress dust-to-gas ratio fluctuations, while for large grains (sizes $\gtrsim 1 \mu \mathrm{m}$ ), Lorentz forces have essentially no effect, trends that are well explained with a simple theory of dust magnetization. In some special intermediate cases, Lorentz forces can enhance dust-gas segregation. Regardless, for the physically expected scaling of dust charge with grain size, we find the most important effects depend on grain size (via the drag equation) with Lorentz forces/charge as a second-order correction. We show that the dynamics we consider are determined by three dimensionless numbers in the limit of weak background magnetic fields: the turbulent Mach number, a dust drag parameter (proportional to grain size) and a dust Lorentz parameter (proportional to grain charge); these allow us to generalize our simulations to a wide range of conditions.
\end{abstract}

Key words: accretion, accretion discs - instabilities - turbulence - planets and satellites: formation - stars: formation - galaxies: formation - cosmology: theory.

\section{INTRODUCTION}

Dust is crucial for a diverse array of phenomena in astrophysics. Dust plays an important direct role in planet and star formation, and also in 'feedback' processes during star cluster and galaxy formation. Dust is also vital for radiative cooling of gas, attenuation and absorption of light in the interstellar medium (ISM) and the evolution of heavy-element abundances and phases in galaxies. It is also a key observational tracer of the ISM in nearby regions and high-redshift galaxies. This broad importance of dust means that it is critical to understand dust dynamics and grain clustering, in the ISM and star-forming regions.

It has long been known that dust grains do not necessarily move with gas in astrophysical fluids. In protoplanetary discs, in particular, a wide variety of conditions have been identified where different fluid conditions can produce orders-of-magnitude variations in the local dust-to-gas ratio, including 'pressure' traps, local 'vortex traps' or 'turbulent concentration' in turbulent discs, the streaming

^E-mail: hlee2@mit.edu instability, 'zonal flows' in magnetically active discs and more (see e.g. Bracco et al. 1999; Cuzzi et al. 2001; Youdin \& Goodman 2005; Johansen \& Youdin 2007; Carballido, Stone \& Turner 2008; Bai \& Stone 2010a,b; Pan et al. 2011; Dittrich, Klahr \& Johansen 2013; Jalali 2013; Hopkins 2014a). Large fluctuations in the density of aerodynamic particles relative to gas have also long been observed in terrestrial turbulence (Squires \& Eaton 1991; Fessler, Kulick \& Eaton 1994; Rouson \& Eaton 2001; Gualtieri, Picano \& Casciola 2009; Monchaux, Bourgoin \& Cartellier 2010).

More recently, several studies have suggested that dust grains in GMCs or neutral galactic discs should exhibit similar fluctuations (Padoan et al. 2006; Hopkins 2014b; Hopkins \& Conroy 2017) in terms of the aerodynamic drag equations, a grain of diameter $\sim 0.1-1 \mu \mathrm{m}$ in a typical GMC is analogous to a metre-sized boulder in a protoplanetary disc. And observations have identified smallscale $(\sim 0.01-1 \mathrm{pc})$ fluctuations in the local dust-to-gas ratio of large grains in a number of nearby molecular clouds (Thoraval, Boisse \& Duvert 1997; Thoraval, Boissé \& Duvert 1999; Abergel et al. 2002; Flagey et al. 2009; Boogert et al. 2013). Across different regions in the ISM, variations in extinction curves and emission/absorption features similarly suggest there may be large fluctuations in the 
relative abundance of large grains (Miville-Deschênes et al. 2002; Gordon et al. 2003; Dobashi et al. 2008; Paradis et al. 2009). The solar neighbourhood, in particular, appears to exhibit an anomalous large-grain abundance (Krüger et al. 2001; Meisel, Janches \& Mathews 2002; Frisch \& Slavin 2003; Altobelli, Grün \& Landgraf 2006; Altobelli et al. 2007; Poppe et al. 2010). And Hopkins (2014b) suggested that this could explain some (but not all) of the variations in abundances observed within some star massive clusters. ${ }^{1}$

But there are some important differences between dust dynamics in GMCs, as compared to the more well-studied terrestrial and planetary disc cases: most obviously, that the turbulence in GMCs is highly supersonic, approximately isothermal (because the gas is rapidly-cooling), magnetized and self-gravitating. Hopkins \& Lee (2016, hereafter Paper I) presented a first numerical study of dust as aerodynamic particles under these conditions, and showed that indeed similar, dramatic fluctuations are expected in supersonic, isothermal, magnetohydrodynamic (MHD) turbulence, on scales that could be important for star formation, dust grain growth and a wide variety of other phenomena. However, that study considered only neutral dust grains - i.e. while the gas was magnetized, the grains felt no Lorentz forces. But real grains in GMCs are expected to be charged, and the Lorentz forces should dominate over aerodynamic (drag) forces for sufficiently small grains, or for large grains in sufficiently low-gas-density regions. This is yet another, perhaps critical, way that dust dynamics are different in GMCs and the ISM from terrestrial or protoplanetary disc turbulence.

In this paper, we therefore extend the study of Paper I, to include explicit, self-consistent Lorentz forces on grains, with realistic charges. We will show this does indeed have effects in the regimes expected, but these are generally subdominant to the effects of modest changes in the grain size.

\section{METHODS}

The methods here exactly follow Paper I with the addition of Lorentz forces, so we briefly summarize here and refer to that paper for details. Our simulations use the code GIZMO (Hopkins 2015). ${ }^{2}$ The gas obeys the equations of ideal MHD with an isothermal equation of state, evolved using the Lagrangian 'MFM' (meshless finitemass) method; extensive tests of the method demonstrating excellent agreement with other well-studied higher order codes on suband supersonic MHD turbulence problems are presented in Hopkins (2015) and Hopkins \& Raives (2016). This is solved in a 3D, periodic box with turbulence driven following Bauer \& Springel (2012), as an Ornstein-Uhlenbeck process with a specified turbulent Mach number and 'natural' (equal) mix of compressive and solenoidal modes. The initial density is uniform and runs are initialized with a uniform magnetic field. In all runs except $\mathrm{n} 1$, this is taken to be small in comparison to the energy of the saturated turbulence; however, it is quickly amplified by field tangling and the small-scale dynamo (Brandenburg \& Subramanian 2005) to reach approximate equipartition with the gas. The quoted values of Alfvénic Mach number $\mathcal{M}_{A}=\mathcal{M} /\left\langle v_{A}^{2}\right\rangle^{1 / 2}$ (where $v_{A}=B / \sqrt{4 \pi \rho}$ ) are measured from the

\footnotetext{
${ }^{1}$ Specifically, Hopkins (2014b) argue grain-gas dynamics may be relevant for certain abundance variations in large, low-density clusters (see e.g. Carretta et al. 2009; Carraro 2014), where the grain-decoupling parameter $\alpha$ (introduced below) is maximized, as opposed to low-mass clusters which appear to exhibit smaller abundance spreads (Pancino et al. 2010; Bragaglia et al. 2012; Carrera \& Martinez-Vazquez 2013).

${ }^{2}$ A public version of this code is available at: http://www.tapir.caltech. edu/phopkins/Site/GIZMO.html.
}

saturated state, and increase with $\mathcal{M}$ as expected (Federrath et al. 2011). All statistical quantities given in Table 1 are measured after the turbulence has saturated.

Dust is represented as a population of tracer particles integrated on-the-fly through the fluid (representing grains of fixed size), with the equation of motion (EOM; Draine \& Salpeter 1979):

$$
\begin{aligned}
& \frac{\mathrm{d} \boldsymbol{u}_{\mathrm{d}}}{\mathrm{d} t}=-\frac{\boldsymbol{u}_{\mathrm{d}}-\boldsymbol{u}_{\mathrm{gas}}}{t_{\mathrm{s}}}+\frac{Z_{\mathrm{d}} \boldsymbol{e}}{m_{\mathrm{d}} c}\left(\boldsymbol{u}_{\mathrm{d}}-\boldsymbol{u}_{\mathrm{gas}}\right) \times \boldsymbol{B} \\
& t_{\mathrm{s}} \equiv \frac{\pi^{1 / 2}}{2 \sqrt{2}}\left(\frac{\bar{\rho}_{\mathrm{d}} a_{\mathrm{d}}}{c_{\mathrm{s}} \rho_{\mathrm{gas}}}\right)\left(1+\left|\frac{3 \pi^{1 / 2}}{8 \sqrt{2}} \frac{\boldsymbol{u}_{\mathrm{d}}-\boldsymbol{u}_{\mathrm{gas}}}{c_{\mathrm{s}}}\right|^{2}\right)^{-1 / 2},
\end{aligned}
$$

where $\mathrm{d} / \mathrm{d} t$ is a Lagrangian derivative, $\boldsymbol{u}_{\mathrm{d}}, \boldsymbol{u}_{\text {gas }}$ are the grain and gas velocity, $c_{\mathrm{s}}$ and $\rho_{\text {gas }}$ are the isothermal sound speed and density of the gas, $\bar{\rho}_{\mathrm{d}} \sim 2.4 \mathrm{~g} \mathrm{~cm}^{-3}$ is the internal (material) grain density (Draine 2003) $)^{3}, a_{\mathrm{d}} \sim 0.001-1 \mu \mathrm{m}$ is the radius of single grain, $Z_{\mathrm{d}} e$ is the grain charge, $c$ is the speed of light and $m_{\mathrm{d}}=(4 \pi / 3) \bar{\rho}_{\mathrm{d}} a_{\mathrm{d}}^{3}$ is the grain mass. The first term is the drag term, with 'stopping time' $t_{\mathrm{s}}$, the second term is the Lorentz term in a magnetic field $\boldsymbol{B}$. The gas quantities are kernel-interpolated to the dust grain locations and the dust trajectories are integrated using a semi-implicit leapfrog scheme, as detailed in Paper I. In addition to the Courant condition for neutral dust time-integration given in Paper I (section 2.2), stably integrating the Lorentz equation requires the dust timesteps be less than a small fraction (we take 10 per cent) of the Larmor time $m_{\mathrm{d}} c /\left(Z_{\mathrm{d}} e|\boldsymbol{B}|\right)$.

Radiation pressure, gas self-gravity, dust-dust collisions and back-reaction of dust grains on gas are not included here: arguments in section 2.4 of Paper I show these all tend to be highly subdominant to drag and Lorentz forces in the systems we simulate here (in clouds of relatively low metallicity). Introducing them also breaks the scale-free nature of the problem here, necessitating a broader simulation survey and a different type of simulation (following star formation self-consistently, for example). However, such effects can be important in specific situations (e.g. dust back reaction on the gas in the highest-density regions, radiation pressure on dust near massive stars) and in future work we will examine some of these effects in more detail.

Without loss of generality, we work in code units where $c_{\mathrm{s}}=1$, the box length $L_{\mathrm{box}}=1$, and the box gas mass $M_{\mathrm{box}} \equiv\left\langle\rho_{\mathrm{gas}}\right\rangle L_{\mathrm{box}}^{3}=1$; equation (1) then becomes

$\alpha \frac{\mathrm{d} \tilde{\boldsymbol{u}}_{\mathrm{d}}}{\mathrm{d} \tilde{t}}=-\Delta \tilde{\boldsymbol{u}} \tilde{\rho}_{\mathrm{gas}} f(\Delta \tilde{\boldsymbol{u}})+\phi \Delta \tilde{\boldsymbol{u}} \times \tilde{\boldsymbol{B}}$

$\Delta \tilde{\boldsymbol{u}} \equiv \tilde{\boldsymbol{u}}_{\mathrm{d}}-\tilde{\boldsymbol{u}}_{\mathrm{g}}, f^{2} \equiv \frac{8}{\pi}+\frac{9}{16}|\Delta \tilde{\boldsymbol{u}}|^{2}$

$\alpha \equiv \frac{\bar{\rho}_{\mathrm{d}} a_{\mathrm{d}}}{\left\langle\rho_{\mathrm{gas}}\right\rangle L_{\mathrm{box}}} \approx 20 \frac{a_{\mathrm{d}}}{\mu \mathrm{m}} \frac{\mathrm{pc}}{L_{\mathrm{box}}} \frac{\mathrm{cm}^{-3}}{\left\langle n_{\mathrm{gas}}\right\rangle} \frac{\bar{\rho}_{\mathrm{d}}}{2.4 \mathrm{~g} \mathrm{~cm}^{-3}}$

$\phi \equiv \frac{3 Z_{\mathrm{d}} e}{4 \pi c a_{\mathrm{d}}^{2}\left\langle\rho_{\mathrm{gas}}\right\rangle^{1 / 2}} \approx 0.2 Z_{\mathrm{d}}\left(\frac{\mu \mathrm{m}}{a_{\mathrm{d}}}\right)^{2}\left(\frac{\mathrm{cm}^{-3}}{\left\langle n_{\mathrm{gas}}\right\rangle}\right)^{1 / 2}$,

\footnotetext{
${ }^{3}$ Of course, the internal material properties of large dust grains, which may be complicated aggregates with ice mantles, are quite uncertain (although see Draine 2003, who argues non-compactness would alter densities by only a small factor in ISM dust). For this reason and others we will define the grain dynamics in terms of dimensionless quantities ( $\alpha$ and $\phi$ below) which can then be trivially re-scaled for any material density.
} 
Table 1. 3D simulations of dust-gas dynamics in this paper.

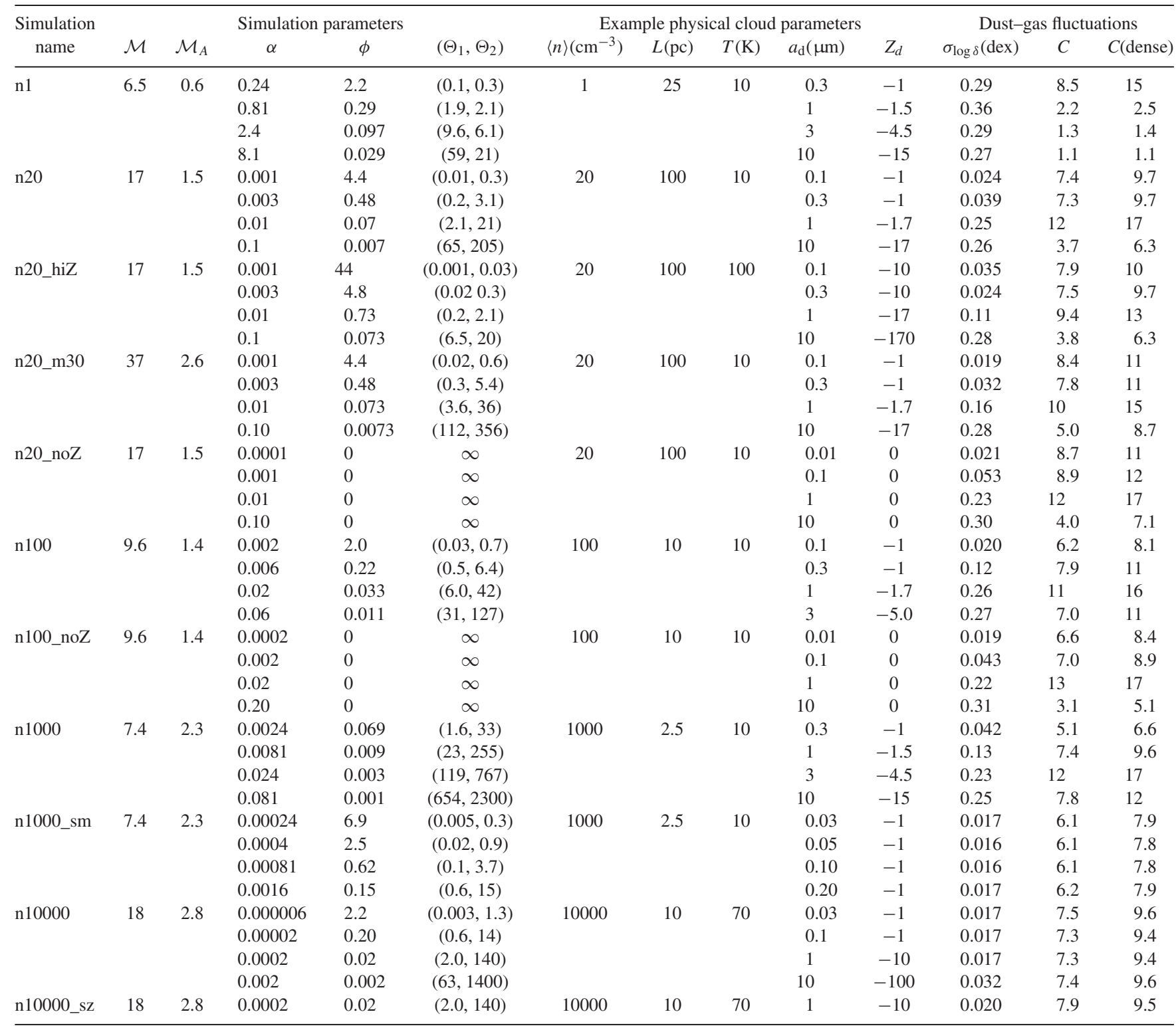

List of simulations analysed in the text: each is a 3D MHD simulation with driven isothermal turbulence, and grains of various sizes with dynamics determined by drag and Lorentz forces (equation 1). Columns (2)-(6) show various dimensionless parameters for each simulation: $\mathcal{M}$, steady-state turbulent rms Mach number on the box scale (set by driving routines and mass-weighted); $\mathcal{M}_{A}$, steady-state Alfvénic Mach number $\mathcal{M}_{A}=\mathcal{M} / v_{A}$ (where $v_{A}$ is the mass-weighted rms Alfvén speed); $\alpha$, grain drag/size parameter (equation 5); $\phi$, Lorentz parameter (equation 6); $\left(\Theta_{1}, \Theta_{2}\right)$, dust magnetization parameters, which provide an estimate whether dust trajectories are significantly affected by the magnetic field (see Section 3 and equation 10). These dimensionless parameters allow us to study a broad range of systems with fewer simulations and isolate the key controlling parameters. In other words, even with different physical parameters, two clouds at the same $\mathcal{M}, \mathcal{M}_{A}, \alpha$ and $\phi$ will exhibit the same physics. Columns (7)-(10) give an example of one specific set of physical cloud parameters which could produce the given $\mathcal{M}, \alpha, \phi$ - here we select a typical isothermal temperature $T$ for cold molecular gas in local GMCs (10 K) or warm gas in starburst regions $(70 \mathrm{~K})$, a box size $L_{\text {box }}$ and density $\left\langle n_{\text {box }}\right\rangle$ roughly on the observed linewidth-size relation and mass-size relations from Bolatto et al. (2008), a set of physical (large) grain sizes $a_{d}$, and grain charges $Z_{d}$ determined from Draine \& Sutin (1987) (see text). Columns (10)-(13) summarize the (time-averaged) dust-to-gas fluctuations measured from simulation in saturated steady-state: $\sigma_{\log \delta}$ is the logarithmic dispersion in $\delta\left(\sigma_{\log \delta}^{2} \equiv\left\langle\left(\log _{10} \delta\right)^{2}\right\rangle-\left\langle\log _{10} \delta\right\rangle^{2}\right)$ from Fig. 3, where the local dust-to-gas ratio $\delta \equiv\left(n_{\text {dust }} / n_{\text {gas }}\right) /\left(\left\langle n_{\text {dust }}\right\rangle /\left\langle n_{\text {gas }}\right\rangle\right) . C \equiv\left\langle n_{\text {dust }}^{2}\right\rangle /\left\langle n_{\text {dust }}\right\rangle^{2}$ is the volume-weighted dust clumping factor. $C($ dense $)$ is $C$ measured only in the dense $\left(n_{\text {gas }}>\left\langle n_{\text {gas }}\right\rangle\right)$ gas. Note that clumping factors quoted in Paper I were accidentally weighted incorrectly, making them larger by a factor $\sim 5$.

where the tilde-superscript $\tilde{x}$ denotes the value of $x$ in code units. ${ }^{4}$ Here, $\alpha$ is the dimensionless 'drag' or 'size' parameter from Paper I

\footnotetext{
${ }^{4}$ We will, for convenience, define the number density $\left\langle n_{\text {gas }}\right\rangle \equiv\left\langle\rho_{\text {gas }}\right\rangle / \mu m_{\mathrm{p}}$ with $\mu=2.3$ (appropriate for solar metallicity, molecular gas), but $\mu$ never enters the dynamics.
}

(smaller $\alpha$ means stronger gas-grain coupling), while the 'Lorentz parameter' $\phi$ governs the effect of the Lorentz force.

In isothermal MHD turbulence starting from trace magnetic fields, the statistics of $\tilde{\boldsymbol{u}}_{\mathrm{g}}$ and the saturated $\tilde{\boldsymbol{B}}$ are determined by the box-averaged Mach number $\mathcal{M} \equiv\left\langle\left|\boldsymbol{u}_{\text {gas }}\right|^{2}\right\rangle^{1 / 2} / c_{\mathrm{s}}=\left\langle\left|\tilde{\boldsymbol{u}}_{\text {gas }}\right|^{2}\right\rangle^{1 / 2}$ (the statistics of the forcing can also be important; Federrath 2013). 
Thus, aside from the initial mean field (energetically important in the saturated state only for run ' $n 1$ '), the saturated dynamics of the problem are governed by $\mathcal{M}, \alpha$ and $\phi$, greatly simplifying our parameter survey. All runs here use $256^{3}$ gas and $2 \times 256^{3}$ dust particles and a variety of convergence studies in Paper I (appendix B, fig. B1) demonstrate that this is sufficient for converged results in quantities studied here. Table 1 presents the list of simulations we study. For each run, we select $\mathcal{M}, \alpha, \phi$ by initially selecting a representative temperature $T$ for cold, molecular gas and box size $L_{\mathrm{box}}$, then estimating the Mach number and mean density corresponding to observed GMCs on the linewidth-size and mass-size relations from Bolatto et al. (2008). We then populate the box with four (relatively large $)^{5}$ physical grain sizes $(\alpha)$ and determine the charge $Z_{\mathrm{d}}$ (and $\phi$ ) of each following Draine \& Sutin $(1987)^{6}:\left\langle Z_{\mathrm{d}}\right\rangle=-1 /\left(1+0.037 \tau^{-1 / 2}\right)-2.5 \tau$, where $\tau \equiv a_{\mathrm{d}} k T / e^{2}$. For comparison, we consider some cases with $Z_{\mathrm{d}}=0$ (no Lorentz forces; our 'noZ' runs) or $10 \times$ larger charge (our 'hiZ' runs) - this crudely corresponds to the expected values if the gas were $10 \times$ hotter (but retained the same Mach number). One run, ' $n 10000 \_s z$ ' is run with twice as many gas elements and all dust grains the same size to verify convergence; we find the statistics are nearly identical to our 'standard' run.

\section{DUST MAGNETIZATION}

In this section, we estimate the influence of the magnetic field on dust-grain dynamics. A convenient way to parametrize this is through the ratio of the dust gyroradius $r_{\mathrm{gy}, \mathrm{d}}$ to its free-streaming length in the gas $L_{\text {stream. }}$. If $r_{\mathrm{gy}, \mathrm{d}}>L_{\mathrm{stream}}$, then the dust is effectively unmagnetized (it is stopped before undergoing a gyro-orbit), while if $r_{\mathrm{gy}, \mathrm{d}}<L_{\text {stream }}$ the magnetic field will have a strong influence on dust dynamics (Lazarian \& Yan 2002). Here, we estimate $r_{\mathrm{gy}, \mathrm{d}} / L_{\mathrm{stream}}$ assuming a basic supersonic turbulence model. Although qualitative, these arguments aid in understanding when dust charging should significantly modify its density distribution. As discussed below (see Section 4), we find reasonable agreement between the theory and simulation results.

The dust free-streaming length may be estimated as $L_{\text {stream }} \sim$ $\left\langle\left|\boldsymbol{u}_{\mathrm{d}}-\boldsymbol{u}_{\text {gas }}\right|\right\rangle t_{\mathrm{s}}$, where the stopping time $t_{\mathrm{s}}$ is given by equation (2) and the relative velocity $\left\langle\left|\boldsymbol{u}_{\mathrm{d}}-\boldsymbol{u}_{\text {gas }}\right|\right\rangle$ may be estimated as the 'eddy velocity' $v_{\lambda} \sim\left\langle\left|\boldsymbol{u}_{\text {gas }}(\boldsymbol{r}+\lambda)-\boldsymbol{u}_{\text {gas }}(\boldsymbol{r})\right|\right\rangle$ (with $\left.\lambda=|\lambda|\right)$ of the turbulence on scale $\lambda=L_{\text {stream }}{ }^{7}$ We then assume the standard hydrodynamic velocity scalings $v_{\lambda} \sim \mathcal{M} c_{\mathrm{s}}\left(\lambda / L_{\mathrm{box}}\right)^{1 / 2}$ for $v_{\lambda}>c_{\mathrm{s}}$; $v_{\lambda} \sim c_{\mathrm{s}}\left(\lambda / R_{\text {sonic }}\right)^{1 / 3}$ for $v_{\lambda}<c_{\mathrm{s}}$, where $R_{\text {sonic }} \sim L_{\text {box }} \mathcal{M}^{-2}$ is the scale at which the turbulence transitions from supersonic to subsonic. This scaling assumes that the influence of the magnetic field on the flow should be relatively unimportant until at, or below, the

\footnotetext{
${ }^{5}$ We focus on large grains $a_{\mathrm{d}} \sim 0.1-10 \mu \mathrm{m}$ because (1) these contain most of the dust mass, and (2) smaller grains are tightly-coupled to the gas and therefore exhibit less extreme dust-to-gas fluctuations.

${ }^{6}$ Draine \& Sutin (1987) estimate grain charges based on a pure collisional model for large grains and a polarization model for small grains. Shull (1978) show that accounting for higher-order effects can lower the charge by a factor $\sim 2$ when the dust-gas motion is highly supersonic. Since this is uncertain, we remind the reader that the parameter $\phi$ is what actually enters the dynamical equations solved here.

${ }^{7}$ This is because velocities on smaller scales do not strongly perturb the dust, while those on larger scales simply advect dust and gas together; see Voelk et al. (1980), Lazarian \& Yan (2002) and Paper I.
}

subsonic scales $\left(\lambda \leq R_{\text {sonic }}\right){ }^{8}$ Otherwise - i.e. in the case of strong mean fields - the turbulence would be Alfvénic in character and anisotropic at large scales (Lithwick \& Goldreich 2001; Cho \& Lazarian 2003). Our analysis is thus restricted to turbulence where $\mathcal{M}_{A}>1$ and the field is tangled on supersonic scales (this is the opposite regime to Yan et al. 2004). This appears to be satisfied for most of the simulations detailed in Table 1 (an exception is ' $n 1$ ', which has a relatively strong mean field).

Assuming the force on dust from the magnetic field will not strongly alter the streaming length, one can estimate (see Paper I)

$$
\frac{L_{\text {stream }}}{L_{\text {box }}} \sim\left\{\begin{array}{ll}
\alpha\left(\frac{n_{\text {gas }}}{\left\langle n_{\text {gas }}\right\rangle}\right)^{-1} & L_{\text {stream }}>R_{\text {sonic }} \\
\alpha^{3 / 2} \mathcal{M}\left(\frac{n_{\text {gas }}}{\left\langle n_{\text {gas }}\right\rangle}\right)^{-3 / 2} & L_{\text {stream }}<R_{\text {sonic }}
\end{array} .\right.
$$

The transition between the two regimes occurs when $n_{\text {gas }} /\left\langle n_{\text {gas }}\right\rangle \sim$ $\alpha \mathcal{M}^{2}$. Noting that the density contrast in an isothermal shock is $n_{\text {gas }} /\left\langle n_{\text {gas }}\right\rangle \sim \mathcal{M}^{2}$ (Passot \& Vázquez-Semadeni 1998; Konstandin et al. 2012), we see that there are three regimes (see Paper I, section 3.1 for further discussion): (i) if $\alpha \gtrsim 1, L_{\text {stream }}>R_{\text {sonic }}$ everywhere (including the shocks) and the dust is weakly coupled to the gas; (ii) if $\mathcal{M}^{-2} \lesssim \alpha \lesssim 1$ the dust is trapped in the highest density shocks but can cluster on scales larger than the sonic length; (iii) if $\alpha \ll \mathcal{M}^{-2}$ the dust is strongly coupled to the gas down to scales below the sonic length.

To estimate the ratio $r_{\mathrm{gy}, \mathrm{d}} / L_{\mathrm{stream}}$, we assume $B / \rho_{\mathrm{gas}}^{1 / 2} \sim$ $c_{\mathrm{s}} \mathcal{M} / \mathcal{M}_{A} \sim$ constant, everywhere in the turbulence. This estimate is supported by observations (Crutcher 1999; Crutcher et al. 2010) and numerical simulations (Banerjee et al. 2009; Burkhart et al. 2009; Molina et al. 2012) in the regime of interest where the fields dynamically unimportant on supersonic scales (i.e. we again require $\left.\mathcal{M}_{A}>1\right) .{ }^{9}$ Note that the dust feels the total 'large-scale' magnetic field (in contrast to the velocity field, where only $v_{\lambda}$ is important), so we do not need the magnetic field spectrum. Using the dust gyroradius $r_{\mathrm{gy}, \mathrm{d}}=m_{\mathrm{d}} c\left|\boldsymbol{u}_{\mathrm{d}}\right| / Z_{\mathrm{d}} e B$ and equations (5)-(6), one obtains

$$
\frac{r_{\text {gy }, \mathrm{d}}}{L_{\text {stream }}} \sim \frac{\alpha}{\phi}\left(\frac{n_{\text {gas }}}{\left\langle n_{\text {gas }}\right\rangle}\right)^{-1 / 2}\left(\frac{L_{\text {stream }}}{L_{\text {box }}}\right)^{-1} v_{L_{\text {stream }}} \frac{\mathcal{M}_{A}}{\mathcal{M}} .
$$

Inserting equation (7) into equation (8) leads to the estimate

$$
\frac{r_{\text {gy }, \mathrm{d}}}{L_{\text {stream }}} \sim\left\{\begin{array}{ll}
\frac{\alpha^{1 / 2}}{\phi} \mathcal{M}_{A} & L_{\text {stream }}>R_{\text {sonic }} \\
\frac{1}{\phi}\left(\frac{n_{\text {gas }}}{\left\langle n_{\text {gas }}\right\rangle}\right)^{1 / 2} \frac{\mathcal{M}_{A}}{\mathcal{M}} & L_{\text {stream }}<R_{\text {sonic }}
\end{array} .\right.
$$

Equation (9) illustrates that the dust magnetization, $r_{\mathrm{gy}, \mathrm{d}} / L_{\mathrm{stream}}$, is governed by the parameters

$\Theta_{1}=\frac{\alpha^{1 / 2}}{\phi} \mathcal{M}_{A}$ and $\Theta_{2}=\frac{1}{\phi} \mathcal{M}_{A}$.

\footnotetext{
${ }^{8}$ Note that we have also assumed a subsonic scaling $v_{\lambda} \sim \lambda^{1 / 3}$, which only holds perpendicular to the magnetic field in magnetized turbulence (Goldreich \& Sridhar 1995; Maron \& Goldreich 2001). This estimate is more appropriate than the parallel scaling $v_{\lambda_{\|}} \sim \lambda_{\|}^{1 / 2}$ when the dust gyroradius is larger than the smallest perpendicular scales, since the dust will not be perfectly tied to the field lines (see Lazarian \& Yan 2002 and Yan et al. 2004 for further discussion).

${ }^{9}$ Crutcher et al. (2010) report a lower density bound, below which the field and density are uncorrelated. This might be expected as the turbulence transitions into an Alfvénic regime, but we ignore this possible change in scaling here for simplicity.
} 
Recalling that the transition between the $L_{\text {stream }}>R_{\text {sonic }}$ and $L_{\text {stream }}<R_{\text {sonic }}$ regimes occurs at $n_{\text {gas }} /\left\langle n_{\text {gas }}\right\rangle \sim \alpha \mathcal{M}^{2}$, and noting that $r_{\mathrm{gy}, \mathrm{d}} / L_{\text {stream }}$ increases monotonically with density, we see that there are three regimes:

$\Theta_{1}>1$ - unmagnetized: The dust is always 'unmagnetized' $\left(r_{\mathrm{gy}, \mathrm{d}}>L_{\text {stream }}\right.$ over all scales $)$.

$\Theta_{1}<1$ and $\Theta_{2}>1-$ mixed: The dust is magnetized at low gas densities $n_{\text {gas }}<n_{\text {g,crit }}$, but switches to being unmagnetized $\left(r_{\mathrm{gy}, \mathrm{d}}>L_{\mathrm{stream}}\right)$ as it streams into high-density regions $n_{\mathrm{gas}}>n_{\mathrm{g}, \mathrm{crit}}$. The critical gas density that governs the change is

$\frac{n_{\text {g,crit }}}{\left\langle n_{\text {gas }}\right\rangle} \sim \phi^{2}\left(\frac{\mathcal{M}}{\mathcal{M}_{A}}\right)^{2}$.

$\Theta_{1}<1$ and $\Theta_{2}<1-$ magnetized: The dust is always magnetized ( $r_{\mathrm{gy}, \mathrm{d}}<L_{\text {stream }}$ over all scales). This is because the density in the shocked regions $n_{\text {gas }} /\left\langle n_{\text {gas }}\right\rangle \sim \mathcal{M}^{2}$ is still not sufficiently high to make $r_{\mathrm{gy}, \mathrm{d}}>L_{\text {stream }}$.

The simulations presented below cover each of these regimes [see $\left(\Theta_{1}, \Theta_{2}\right)$ column of Table 1]. Note that our discussion here has been intended to estimate when the magnetic field is important for the dust, as opposed to the influence of the magnetic field on the dust distribution (this is discussed in more detail in the next section).

\section{RESULTS}

In Figs 1-2, we show the bivariate distribution of dust and gas densities in some representative simulations. ${ }^{10}$ Here and throughout this paper, all distribution functions are dust-mass weighted. In Fig. 1, we see the effects of increasing $\alpha$ (grain size). As described above (see equation 7) and in Paper I, small grains $\left(\alpha \ll \mathcal{M}^{-2}\right)$ are tightly coupled to gas, very large grains $(\alpha>1)$ are spread closer to uniformly and weakly-coupled to the gas, and grains with intermediate $\alpha\left(\mathcal{M}^{-2} \lesssim \alpha \lesssim 1\right)$ produce interesting dust-gas distributions. ${ }^{11}$ This behaviour is similar to that seen in Paper I without Lorentz forces; for a more detailed analysis of the gas-density dependence of fluctuations, and their dependence on spatial scale (power spectra/correlation functions), we refer interested readers to Paper I.

Fig. 2 shows the effect of adding Lorentz forces at two grain sizes for three different levels of dust charge (no charge, 'n20_noZ'; 'standard' charge, 'n20'; and $10 \times$ charge, 'n20_hiZ'). The illustrated dust-gas distributions broadly follow our expectations based on the theory of dust magnetization in Section 3. Small grains $(\alpha=0.001)$

\footnotetext{
${ }^{10}$ The dust and gas densities are determined in post-processing as in Paper I, using an adaptive kernel density estimator enclosing the nearest $\approx 64$ particles at the location of every dust particle to evaluate the dust density $n_{\text {dust }}\left(a_{\mathrm{d}}\right)$ (counting species only of the same size) and $n_{\text {gas }}$ at the same location. We have verified that the results are insensitive to the number of neighbours or shape of the kernel. After the first few turbulent crossing times the simulation reaches steady-state and there are no significant trends with time, so we simply average all snapshots together after this time.

${ }^{11}$ Note, in Fig. 1, for the same grain size, the fluctuations at a given physical density (say, $n_{\text {gas }} \sim 10^{3} \mathrm{~cm}^{-3}$ ), in the 'n20' run (at $50 \times$ mean density) are much larger than the fluctuations at the same mean density in the 'n1000' or ' $n 10000$ ' run. This is discussed in Paper I, Hopkins (2014b) and Hopkins \& Conroy (2017). Basically, because the high-density regions in the lower-mean-density box form from a wide range of progenitor regions with lower pre-shock/compression density (hence weaker dust-gas coupling), their dust-to-gas ratio fluctuations are 'seeded' in these progenitors. Once a region locally becomes sufficiently dense that grains are tightly trapped, these are 'frozen in', while new local fluctuations are suppressed.
}

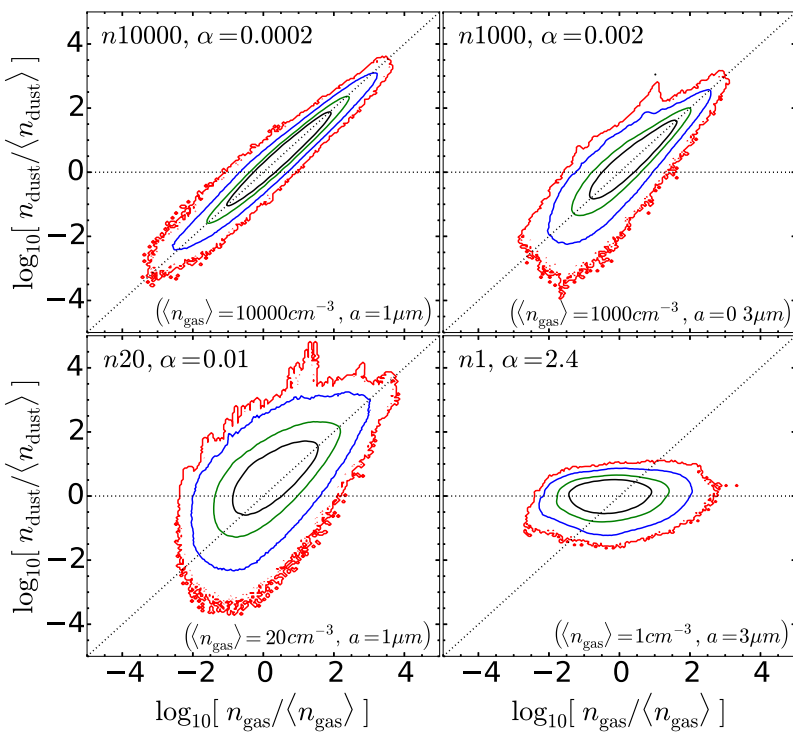

Figure 1. Time-averaged bivariate distribution of dust and gas densities, in representative simulations from Table 1 . We plot iso-density contours at fixed probability density levels $\mathrm{d} P / \mathrm{d} \log n_{\text {gas }} \mathrm{d} \log n_{\text {dust }}=10^{-1}, 10^{-2}, 10^{-4}, 10^{-7}$ (black, green, blue, red, respectively). Dotted lines show $n_{\text {dust }}=\left\langle n_{\text {dust }}\right\rangle$ (dust at constant density) and $n_{\text {dust }}=\left(\left\langle n_{\text {dust }}\right\rangle /\left\langle n_{\text {gas }}\right\rangle\right) n_{\text {gas }}$ (perfect dust-gas coupling, i.e. $\delta=1)$. The simulations shown are chosen to reflect increasing value of the 'drag parameter' $\alpha$ (equation 5), which determines how tightly coupled the dust and gas are. As explained in Section 3, for $\alpha \ll \mathcal{M}^{-2}$, the coupling is near-perfect. For $\alpha \gtrsim 1$, the dust is almost entirely un-correlated with the gas. Intermediate $\alpha$ show dust roughly following gas, $n_{\text {dust }} \propto n_{\text {gas }}$, but with large local fluctuations in $n_{\text {dust }}$ at all $n_{\text {gas }}$. Note that the distribution in $\mathrm{n} 10000$ is likely affected by Poisson noise and may be even more tightly coupled to the gas than it appears: its distribution resembles the limiting $\alpha \ll 1$ case (where the dust is perfectly coupled to the gas) as shown in Paper I, fig. C1.

in both 'n20' and 'n20_hiZ' are magnetized everywhere in the gas $\left(\Theta_{1}<1, \Theta_{2}<1\right)$, and indeed the dust-gas distributions are quite different to the uncharged case ('n20_noZ'), with tighter coupling of the dust to the gas. In contrast, the large grains $(\alpha=0.01)$ are either unmagnetized (for standard grain charge, 'n20') or in the 'mixed' regime (for $10 \times$ charge, 'n20_hiZ'). In agreement with the theory, the standard-charge ('n20') distribution looks similar to the uncharged case, while the 'n20_hiZ' distribution is similar at high-gas densities (where the grains are unmagnetized) but exhibits stronger dust-gas coupling at low-gas densities (where the grains are magnetized). For the parameters of this simulation ('n20_hiZ' $\alpha=0.01$ ), the critical gas density $n_{\mathrm{g}, \text { crit }}$ governing the change from magnetized to unmagnetized dust [see equation (11)] is $n_{\mathrm{g}, \text { crit }} /\left\langle n_{\text {gas }}\right\rangle \approx 75$, which is consistent with what is observed in Fig. 2 (of course, the change is gradual and the theory heuristic, so we should not expect obvious quantitative agreement)

The dust-to-gas ratio $\delta \equiv\left(n_{\text {dust }} / n_{\text {gas }}\right) /\left(\left\langle n_{\text {dust }}\right\rangle /\left\langle n_{\text {gas }}\right\rangle\right)$ (i.e. integrating out one dimension from Figs $1-2)$ is an interesting quantity for both practical application to GMCs and for theory. We illustrate its distribution in Fig. 3 for each of the 'standard-charge' simulations ('n1', 'n20', 'n100', 'n1000' and 'n10000'). As in Paper I, we find these are approximately log-normal, with power-law tails. More quantitatively, the dispersion of $\delta$ (denoted $\sigma_{\log \delta}$ ) is illustrated in Fig. 4 for all simulations (see also Table 1). There is clearly a strong increase in $\sigma_{\log \delta}$ with $\alpha$-i.e. with larger $a_{\mathrm{d}}$ and smaller $\left\langle n_{\text {gas }}\right\rangle$ - particularly around $\alpha \sim 0.005-0.01$ where $\sigma_{\log \delta}$ increases from $\sim 0.05$ to $\sim 0.35 \mathrm{dex}$. This is expected as grains transition from 




Figure 2. Distribution of dust and gas densities (as Fig. 1), for two different grain sizes ( $\alpha=0.001$, left column; $\alpha=0.01$, right column) and otherwise identical parameters. We compare cases with uncharged grains ('n20_noZ'; top row), with 'standard' grain charge ('n20'; middle row) and with ' $10 \times$ ' grain charge ('n20_hiZ'; bottom row); see Table 1. Lorentz forces reduce the fluctuations in $n_{\text {dust }}\left(n_{\text {gas }}\right)$ for small grains, but have weak effects on large grains. This is explained by the dust-magnetization arguments outlined in Section 3. The small grains $(\alpha=0.001)$ are in the magnetized regime $\left(\Theta_{1}<1, \Theta_{2}<1\right)$ in both the 'n20' and 'n20_hiZ' simulations, and so are strongly affected by the magnetic field. In contrast, the larger grains $(\alpha=0.01)$ are unmagnetized $\left(\Theta_{1}>1\right)$ in ' $\mathrm{n} 20$ ', so the dust-gas distribution is similar to that in 'n20_noZ' simulation. The $\alpha=0.01$ grains in 'n20_hiZ' are in the 'mixed' regime $\left(\Theta_{1}<1, \Theta_{2}>1\right)$, which suggests that grains should be magnetized at low-gas densities and unmagnetized at high-gas densities. This idea is supported by the dust-gas distribution (bottom-right panel), which appears similar to that of 'n20' and 'n20_noZ' at high-gas densities, but shows comparatively reduced dust variance at low-gas densities.

being tightly coupled to the gas for $\alpha \ll \mathcal{M}^{-2}$ to uniformly filling the box if $\alpha \gg 1$ (see Paper I and equation 7), and is a more quantitative illustration of the effects shown in Fig. 1. Also note that the 'floor' at $\sigma \sim 0.015-0.02(\alpha \lesssim 0.001)$ is not real, but represents the limitations of our numerical method. ${ }^{12}$ Fig. 4 also serves to illustrate that the effects of dust magnetization on the dispersion of $\delta$ are subdominant to its variation with $\alpha$, although the magnetized cases mostly show slightly lower $\sigma_{\log \delta}$. In other words, the change to the dust-gas distribution with magnetization seen in Fig. 2 causes

${ }^{12}$ As discussed in Paper I (see also Genel et al. 2013), the mismatch between the EOM for grains, where gas quantities are interpolated to the exact grain location, and gas, where the fluxes are calculated from a Riemann problem and averaged over a finite volume, means there will inevitably be some small, purely numerical dust-to-gas fluctuations even when the two should be perfectly-coupled. There we showed $\alpha \lesssim 0.001$ hits this 'floor'. only a small modification to $\sigma_{\log \delta}$ in comparison to the variation with grain size. A more detailed discussion of the non-magnetized scaling is given in Paper I.

It is helpful to examine the changes in dust-to-gas ratio distributions with individual parameters, which is done in Fig. 5. The left-hand panel shows the effects of Mach number $\mathcal{M}$ on the $\delta$ distribution with otherwise equal simulation parameters. We see that the effect on the $\delta$ distribution is minor, even though the logarithmic dispersion in the gas density in the higher- $\mathcal{M}$ run is significantly larger (by $\approx 0.2 \mathrm{dex}$, in agreement with the well-studied Mach number-density variance relation; Konstandin et al. 2012). There is none the less some weak effect of $\mathcal{M}$ on $\delta$ : at the lowest $\alpha$, the tails in $\delta$ are broadened (because dust in the lower- $n_{\text {gas }}$ tails of the gas distribution is, locally, more weakly-coupled), while at large $\alpha$, the distribution actually becomes slightly more narrow (because the grains are already loosely-coupled, this moves the system more towards a 'uniformly mixed' distribution).

In a similar vein, the right-hand panel of Fig. 5 compares simulation with neutral grains ('n20_noZ'), 'standard-charge' grains ('n20'), and 10× charged grains ('n20_hiZ'), keeping all other parameters fixed. This is another way of examining the data shown in Fig. 2. Similar to the discussion above, we see that the small grains $(\alpha=0.001)$ in both the 'n20' and 'n20_hiZ' simulations are quite different to the neutral grains ('n20_noZ'), but similar to each other (aside from an increased dispersion in 'n20_hiZ', perhaps due to resonant acceleration; see below). In contrast, large grains $(\alpha=0.01)$ are similar between the neutral and standard-charge grains ${ }^{13}$ (since these are unmagnetized), while the $10 \times$ charged grains exhibit a substantial decrease in variance compared to the neutral grains because they are magnetized in low-gas-density regions (they are in the 'mixed' regime).

Finally, it is worth commenting on an interesting feature of the $\delta$ distributions in Figs 3 and 5 - the flat, high- $\delta$ tail that appears in some simulations (e.g. $\alpha=0.001$, 'n20'). A comparison with the parameters in Table 1 shows that this exists only for those parameters at which the dust is magnetized $\left(\Theta_{1}<1, \Theta_{2}<1\right)$, while the comparison to an equivalent neutral dust simulation in Fig. 5(b) shows that it is related to the action of the Lorentz force (the tail appears only for charged grains and is stronger in 'n20_hiZ' compared to ' $\mathrm{n} 20$ '). We speculate that this effect may be related to resonant acceleration of dust grains, which can occur when multiples of the dust Larmor frequency match the turnover frequency of the turbulence as seen by the dust (Lazarian \& Yan 2002; Yan \& Lazarian 2003; Yan et al. 2004). The turbulent magnetic field is then stationary in the dust frame and resonantly exchanges energy with the grains through Landau damping and cyclotron damping (as occurs for ions and electrons in weakly collisional plasmas). The higher dust velocities could be particularly important for dust shattering and coagulation (Yan \& Lazarian 2003), but we leave further study of this interesting effect to future work.

\section{CONCLUSIONS}

We study how charged dust grains behave in GMCs by running idealized simulations of isothermal, magnetized, supersonic turbulence, with a population of dust grains of physically interesting

\footnotetext{
${ }^{13}$ There is actually a slight enhancement in variance in ' $\mathrm{n} 20$ ' compared to 'n20_noZ' for large grains. We speculate that this is because the lowest gas density regions, which would have completely de-coupled from the dust, have weak Lorentz coupling and induce some additional dust concentration.
} 


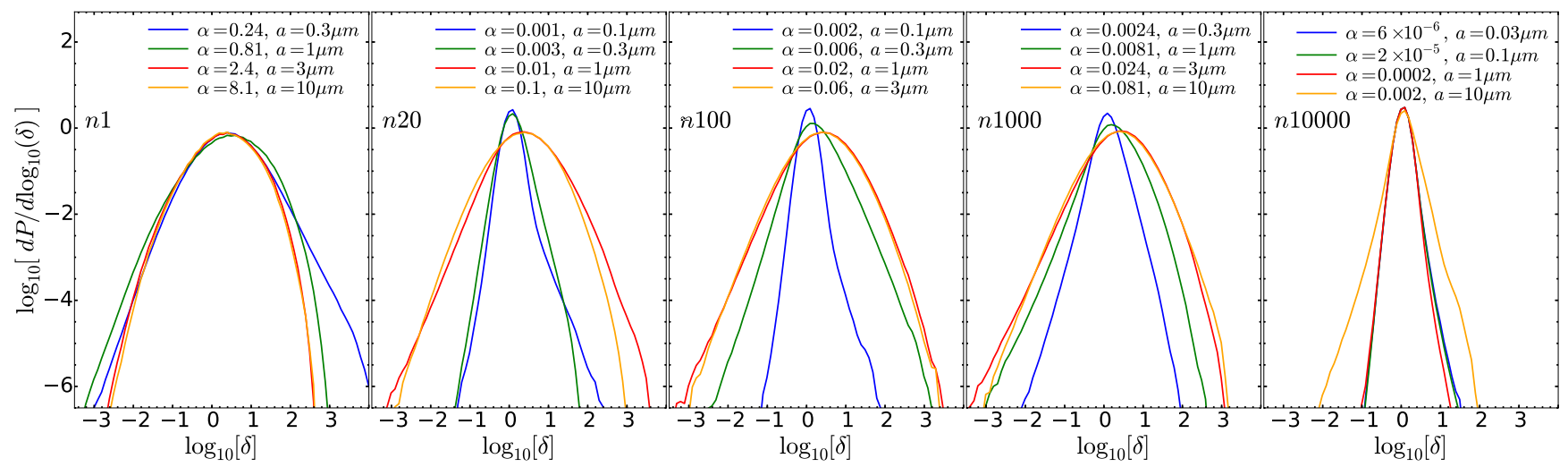

Figure 3. Time-averaged distribution of dust-to-gas ratios $\delta \equiv\left(n_{\text {dust }} / n_{\text {gas }}\right) /\left(\left\langle n_{\text {dust }}\right\rangle /\left\langle n_{\text {gas }}\right\rangle\right)(=1$ at mean densities; see Table 1$)$ in the simulations (with 'normal' Lorentz forces). Each frame shows a different box corresponding to a different mean physical density, and series of different grain drag/size parameters $\alpha$. As expected, $\alpha$ primarily governs the fluctuations: smaller $a_{\mathrm{d}}$ and/or higher $\left\langle n_{\text {gas }}\right\rangle$ reduce the variation $\delta$. As seen in Paper I, the distributions are approximately log-normal in their cores but exhibit strong power-law tails, with slope $\mathrm{d} P / \mathrm{d} \log \delta \propto \delta^{ \pm 2}$ (the steeper falloff at high- $\delta$ in the highest- $\alpha$ runs is closer to $\propto \delta^{-3}$ ).

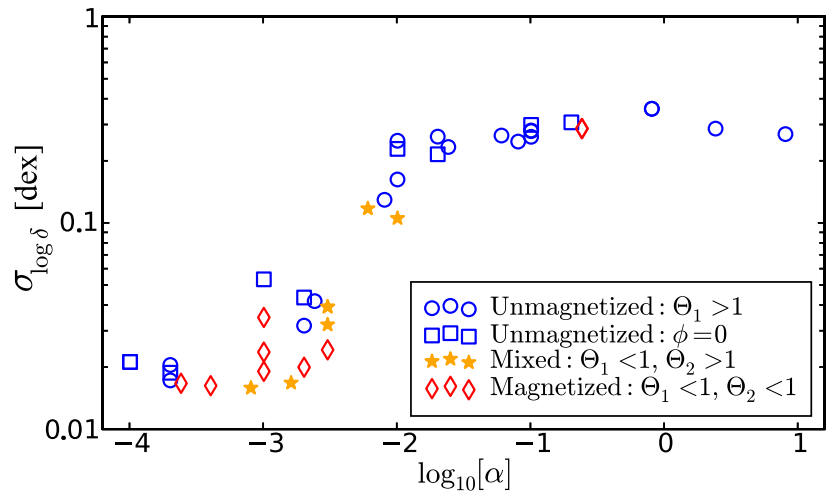

Figure 4. Dispersion $\sigma_{\log \delta}$ of $\log _{10}(\delta)$, for all our simulations, as a function of $\alpha$ and magnetization (see equation 10). Dust-to-gas fluctuations are primarily governed by $\alpha$, and $\sigma_{\log \delta}$ rises steeply from $<0.05$ dex at $\alpha \lesssim$ 0.001 to $\sim 0.3$ dex at $\alpha \gtrsim 0.1$; dust magnetization (and thus $\phi$ ) has only secondary effects. The saturation at high- $\alpha$ is real, since the grains stop feeling drag forces, while the 'floor' in $\sigma$ at $\sim 0.02$ dex is a numerical artefact (see Paper I).

sizes and realistic charge, which experience both drag and Lorentz forces from the gas. We argue that the dynamics are essentially determined by three dimensionless parameters, the turbulent Mach number $\mathcal{M}$, grain size parameter $\alpha \propto a_{\mathrm{d}}$ (equation 5) and Lorentz parameter $\phi \propto Z_{\mathrm{d}} / a_{\mathrm{d}}^{2}$ (equation 6). We show that, when $\mathcal{M} \gg 1$, grain dynamics are strongly governed by the parameter $\alpha$. With small $\alpha \lesssim \mathcal{M}^{-2}$, dust moves tightly with the gas; with large $\alpha \gtrsim$ 1 , grains decouple from the gas and spread uniformly, while intermediate cases (expected for large grains in a wide range of typical GMCs) produce interesting local fluctuations in the dust-to-gas ratio $\delta$, with the logarithmic dispersion in $\delta$ increasing from $\sim 0.05$ to 0.35 dex as $\alpha$ increases. At a given $\alpha$, we show that varying $\mathcal{M}$ (within the range expected in GMCs) has weak effects. Comparing simulations without Lorentz forces, we see the Lorentz forces produce a size-dependent effect: smaller grains (larger $\phi$ ) have their fluctuations suppressed with non-zero $\phi$, while larger grains show weak effects. This can be understood more quantitatively by considering the ratio of dust gyroradius $r_{\mathrm{gy}, \mathrm{d}}$ to dust streaming length $L_{\text {stream }}$, which we examine using the parameters $\Theta_{1}=\alpha^{1 / 2} \mathcal{M}_{A} / \phi$ and $\Theta_{2}=\mathcal{M}_{A} / \phi$ (for $\mathcal{M}_{A} \gtrsim 1$ ). In general, $\Theta_{1} \lesssim 1$ is required for appreciable effects on the dust clustering statistics, which implies that $r_{\mathrm{gy}, \mathrm{d}}<L_{\text {stream }}$ (at least at low densities), meaning the dust dy-
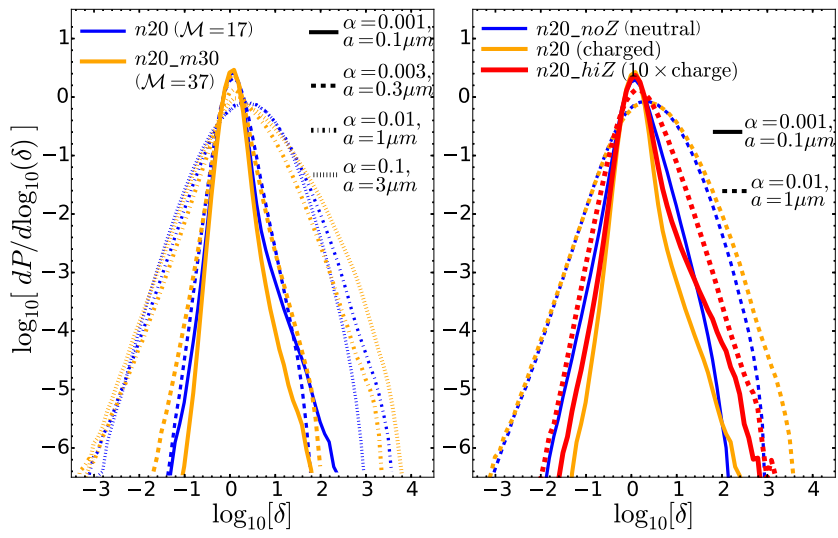

Figure 5. Distribution of $\delta$ (as Fig. 3), for otherwise identical versions of the $\left\langle n_{\text {gas }}\right\rangle=20 \mathrm{~cm}^{-3}$ box (' $\mathrm{n} 20$ '), but varying either the Mach number (lefthand panel) or grain charge/gas temperature (right-hand panel). Increasing $\mathcal{M}$ from $\sim 17$ to $\sim 37$, we see weak effects: for all but the smallest grain sizes the distribution of $\delta$ is broadened, especially at the high- $\delta$ end, as the increased Mach number generates significantly larger variance in the gas density, hence more low-density regions where the grains are effectively de-coupled to the gas. The right-hand panel shows the effect of adding grain Lorentz forces on the $\delta$ distribution. As expected from Fig. 2 (which shows the same simulations), going from neutral dust to normally charged dust (adding Lorentz forces) decreases the variance in $\delta$ for small grains and has a weak effect on large grains. Increasing the grain charge (and $\phi$ ) by a further factor of 10 has a weak additional effect on the small grains (actually slightly increasing the variance in $\delta$ ), but significantly reduces the scatter in the large-grain case. This behaviour is well explained by the dust-magnetization theory outlined in Section 3.

namics are significantly modified by the presence of the magnetic field.

In Paper I (section 4), we discuss implications of partial dust-gas coupling (and variations in local dust-to-gas ratios) for dust formation, extinction and observed dust clustering, cooling and star formation. Because high-density regions can have enhanced/suppressed dust-to-gas ratios in large grains (which contain a large fraction of the metal mass), this can have interesting implications for stellar abundances. Hopkins \& Conroy (2017) use similar simulations, coupled to a specific dust chemistry model, to explore consequences for abundance patterns in metal-poor stars, and suggest that certain observed chemical signatures in these stars may demonstrate 
variable dust-to-gas ratios in their progenitor clouds. Hopkins (2014b) use a simple analytic model to further explore the consequences for stellar abundance variations across present-day star forming clouds. Taking observed scalings of GMC properties (e.g. Bolatto et al. 2008) with size $\sim R_{\mathrm{GMC}}$, they show the critical parameter $\alpha / \mathcal{M}^{-2}$ should scale $\propto R_{\mathrm{GMC}}$ for grains of a fixed size. In physical terms, for sufficiently large clouds $\gtrsim 10-100 \mathrm{pc}$ (for $0.1-1 \mu \mathrm{m}$ grains), $\alpha \gtrsim \mathcal{M}^{-2}$ and grain densities fluctuate on scales greater than the sonic length (the characteristic size of dense star-forming filaments and protostellar cores). All of this work, however, ignored Lorentz forces; our goal here was to explore how this might change the dynamics. Since we find the effects of Lorentz forces are subdominant to grain size variations, none of the key qualitative conclusions from these studies are altered. However, by further suppressing fluctuations in the small-grain regime (while having little effect for large grains), Lorentz forces will make the 'threshold' effect above more dramatic (where fluctuations are unimportant below, but significant above, some characteristic grain/cloud size scale).

A major caveat of this study is that we have considered only the cold, dense ISM in GMCs - the values of $\alpha$ and $\phi$ here are appropriate when $T \lesssim 100 \mathrm{~K}$. It is interesting to ask what happens to dust in the warm-neutral and warm-ionized medium, with $T \sim 10^{3}-10^{4} \mathrm{~K}$; since the equilibrium grain charge (and $\phi$ ) are expected to scale $\propto T$, we expect Lorentz forces to rapidly increase in importance. Unfortunately, the numerical method here (explicitly integrating the Lorentz forces) becomes unacceptably expensive for very large $\phi$ (as the Larmor frequency increases); we are working on a fullyimplicit scheme for integrating the Lorentz term that will allow us to extend our simulations into this regime (also implicit schemes for including dust back-reaction; see Yang \& Johansen 2016). These simulations will also be interesting from a theoretical standpoint, allowing study of the magnetized, high- $\alpha$ region of parameter space that is absent from the simulation set presented in the current work (see, for example, Fig. 4). In the mostly ionized medium, we also need to account for Coulomb interactions, but these primarily manifest as a modest correction to the drag term (Draine \& Salpeter 1979) so their effect should be straightforward to understand and implement numerically. Radiation pressure and dust-collisional dynamics may also modify the conclusions here, especially in the most dense regions where this is relevant for star formation, and we will explore this further in future work.

\section{ACKNOWLEDGEMENTS}

We thank the anonymous referee and Matthew Goodson for a number of useful comments and suggestions. Support for HL \& PFH was provided by NASA ATP Grant NNX14AH35G \& NSF Collaborative Research Grant \#1411920 and CAREER grant \#1455342. JS was funded in part by the Gordon and Betty Moore Foundation through Grant GBMF5076 to Lars Bildsten, Eliot Quataert and E. Sterl Phinney. Numerical calculations were run on Caltech cluster 'Zwicky' (NSF MRI award \#PHY-0960291) \& XSEDE allocation TG-AST130039 supported by the NSF.

\section{REFERENCES}

Abergel A. et al., 2002, A\&A, 389, 239

Altobelli N., Grün E., Landgraf M., 2006, A\&A, 448, 243

Altobelli N., Dikarev V., Kempf S., Srama R., Helfert S., MoragasKlostermeyer G., Roy M., Grün E., 2007, J. Geophys. Res. (Space Physics), 112, 7105
Bai X.-N., Stone J. M., 2010a, ApJS, 190, 297

Bai X.-N., Stone J. M., 2010b, ApJ, 722, L220

Banerjee R., Vázquez-Semadeni E., Hennebelle P., Klessen R. S., 2009, MNRAS, 398, 1082

Bauer A., Springel V., 2012, MNRAS, 423, 3102

Bolatto A. D., Leroy A. K., Rosolowsky E., Walter F., Blitz L., 2008, ApJ, 686, 948

Boogert A. C. A., Chiar J. E., Knez C., Öberg K. I., Mundy L. G., Pendleton Y. J., Tielens A. G. G. M., van Dishoeck E. F., 2013, ApJ, 777, 73

Bracco A., Chavanis P. H., Provenzale A., Spiegel E. A., 1999, Phys. Fluids, 11,2280

Bragaglia A., Gratton R. G., Carretta E., D'Orazi V., Sneden C., Lucatello S., 2012, A\&A, 548, A122

Brandenburg A., Subramanian K., 2005, Phys. Rep., 417, 1

Burkhart B., Falceta-Gonçalves D., Kowal G., Lazarian A., 2009, ApJ, 693, 250

Carballido A., Stone J. M., Turner N. J., 2008, MNRAS, 386, 145

Carraro G., 2014, in Lee H.-W, Kang Y. W., Leung K.-C., eds, ASP Conf. Ser. Vol. 482, The Tenth Pacific Rim Conference on Stellar Astrophysics. Astron. Soc. Pac., San Francisco, p. 245

Carrera R., Martinez-Vazquez C. E., 2013, A\&A, 560, A5

Carretta E. et al., 2009, A\&A, 505, 117

Cho J., Lazarian A., 2003, MNRAS, 345, 325

Crutcher R. M., 1999, ApJ, 520, 706

Crutcher R. M., Wandelt B., Heiles C., Falgarone E., Troland T. H., 2010, ApJ, 725, 466

Cuzzi J. N., Hogan R. C., Paque J. M., Dobrovolskis A. R., 2001, ApJ, 546, 496

Dittrich K., Klahr H., Johansen A., 2013, ApJ, 763, 117

Dobashi K., Bernard J.-P., Hughes A., Paradis D., Reach W. T., Kawamura A., 2008, A\&A, 484, 205

Draine B. T., 2003, ARA\&A, 41, 241

Draine B. T., Salpeter E. E., 1979, ApJ, 231, 77

Draine B. T., Sutin B., 1987, ApJ, 320, 803

Federrath C., 2013, MNRAS, 436, 1245

Federrath C., Chabrier G., Schober J., Banerjee R., Klessen R. S., Schleicher D. R. G., 2011, Phys. Rev. Lett., 107, 114504

Fessler J. R., Kulick J. D., Eaton J. K., 1994, Phys. Fluids, 6, 3742

Flagey N. et al., 2009, ApJ, 701, 1450

Frisch P. C., Slavin J. D., 2003, ApJ, 594, 844

Genel S., Vogelsberger M., Nelson D., Sijacki D., Springel V., Hernquist L., 2013, MNRAS, 435, 1426

Goldreich P., Sridhar S., 1995, ApJ, 438, 763

Gordon K. D., Clayton G. C., Misselt K. A., Landolt A. U., Wolff M. J., 2003, ApJ, 594, 279

Gualtieri P., Picano F., Casciola C. M., 2009, J. Fluid Mech., 629, 25

Hopkins P. F., 2014a, MNRAS, 456, 2383

Hopkins P. F., 2014b, ApJ, 797, 59

Hopkins P. F., 2015, MNRAS, 450, 53

Hopkins P. F., Conroy C., 2017, ApJ, 835, 154

Hopkins P. F., Lee H., 2016, MNRAS, 456, 4174 (Paper I)

Hopkins P. F., Raives M. J., 2016, MNRAS, 455, 51

Jalali M. A., 2013, ApJ, 772, 75

Johansen A., Youdin A., 2007, ApJ, 662, 627

Konstandin L., Girichidis P., Federrath C., Klessen R. S., 2012, ApJ, 761, 149

Krüger H. et al., 2001, Planet. Space Sci., 49, 1303

Lazarian A., Yan H., 2002, ApJ, 566, L105

Lithwick Y., Goldreich P., 2001, ApJ, 562, L279

Maron J., Goldreich P., 2001, ApJ, 554, 1175

Meisel D. D., Janches D., Mathews J. D., 2002, ApJ, 579, 895

Miville-Deschênes M.-A., Boulanger F., Joncas G., Falgarone E., 2002, A\&A, 381, 209

Molina F. Z., Glover S. C. O., Federrath C., Klessen R. S., 2012, MNRAS, 423,2680

Monchaux R., Bourgoin M., Cartellier A., 2010, Phys. Fluids, 22, 103304

Padoan P., Cambrésy L., Juvela M., Kritsuk A., Langer W. D., Norman M. L., 2006, ApJ, 649, 807 
Pan L., Padoan P., Scalo J., Kritsuk A. G., Norman M. L., 2011, ApJ, 740, 6 Pancino E., Carrera R., Rossetti E., Gallart C., 2010, A\&A, 511, A56

Paradis D. et al., 2009, AJ, 138, 196

Passot T., Vázquez-Semadeni E., 1998, Phys. Rev. E, 58, 4501

Poppe A., James D., Jacobsmeyer B., Horányi M., 2010, Geophys. Res. Lett., 37, 11101

Rouson D. W. I., Eaton J. K., 2001, J. Fluid Mech., 428, 149

Shull J. M., 1978, ApJ, 226, 858

Squires K. D., Eaton J. K., 1991, Phys. Fluids A, 3, 1169

Thoraval S., Boisse P., Duvert G., 1997, A\&A, 319, 948
Thoraval S., Boissé P., Duvert G., 1999, A\&A, 351, 1051

Voelk H. J., Jones F. C., Morfill G. E., Roeser S., 1980, A\&A, 85, 316

Yan H., Lazarian A., 2003, ApJ, 592, L33

Yan H., Lazarian A., Draine B. T., 2004, ApJ, 616, 895

Yang C.-C., Johansen A., 2016, ApJS, 224, 39

Youdin A. N., Goodman J., 2005, ApJ, 620, 459

This paper has been typeset from a $\mathrm{T}_{\mathrm{E}} \mathrm{X} / \mathrm{L} \mathrm{T} \mathrm{T} \mathrm{X}$ file prepared by the author. 